\title{
EL ALZHEIMER BORRA LA MEMORIA PERO NO LOS SENTIMIENTOS
}

Alzheimer clears memory, not feelings

O Alzheimer apaga a memória, mas não os sentimentos

\author{
Roberto Fernández Joral \\ Maestro de Educación Primaria, Junta de Castilla y León +34 690083067 y \\ roberto.ferjor@educa.jcyl.es
}

\begin{abstract}
Resumen
El proyecto presenta el cómo desde la educación podemos trabajar por y para un fin social, como es la enfermedad del Alzheimer. Desde la metodología del aprendizaje servicio (ApS) y desde la cooperación, un grupo de alumnos de sexto curso han creado una camiseta para vender en una campaña publicitaria y recaudar fondos para que una asociación de Alzheimer pueda seguir trabajando en esta enfermedad. A la vez de contribuir a este fin social, se han ido trabajando contenidos curriculares y competenciales como: expresión escrita y oral, comprensión lectora, publicidad, herramientas TIC, enfermedades del sistema nervioso, operaciones con números decimales, entre otros. Además de otros de una alta riqueza social al interactuar con personas que padecen esta enfermedad.
\end{abstract}

Palabras clave: Alzheimer; aprendizaje servicio; evaluación formativa; cooperación

\begin{abstract}
The project presents the way we can work to and for a social purpose from education, as it is Alzheimer's disease. From the methodology of learning service (ApS) and from the cooperation mindset, a group of sixth grade students have created a t-shirt designed to be sold in an advertising campaign in order to raise funds so, an Alzheimer association can continue working on this disease. At the same time to contribute to this social purpose, work has been centered on curricular content and competences such as written and oral expression, reading comprehension, advertising, ICT tools, nervous system disorders, operations with decimal numbers and so on, in addition to other socially highly enriching contents to interact with the people who suffer from this disease.
\end{abstract}

Keywords: Alzheimer's Disease; service learning; Formative assessment; cooperation 


\section{Resumo}

O projeto apresenta como é que nós podemos trabalhar desde a educação por e para um fim social, em nosso caso a doença de Alzheimer. Desde uma metodologia da aprendizagem-Serviço (ApS) e cooperação, um grupo de alunos de sexto ano do ensino básico fizeram uma t—shirt para vender numa campanha publicitária e assim obter fundos para que uma associação de Alzheimer continue a trabalhar nesta doença. Ao mesmo tempo, nós trabalhamos conteúdos curriculares e competências: expressão escrita e oral, compreensão leitora, publicidade, ferramentas TIC, doenças do sistema nervoso, cálculo com números decimais entre outros. Além de outros conteúdos com uma maior riqueza social ao interatuar os alunos com pessoas que padecem esta doença. Palavras-chave: Alzheimer, Aprendizagem-Serviço, cooperação e avaliação formativa

\section{Introducción}

El fin social que acontece a la educación pública, necesita de estrategias que fomenten una serie de valores a la vez que se trabajan los contenidos curriculares tal y como apunta Fernández- Enguita (1997). Para ello, el presente proyecto aúna diferentes estrategias metodológicas. Por una parte está bañado por nociones del aprendizaje servicio (en adelante ApS) ayudando no solo a los demás, sino a uno mismo, tal y como destaca Puig (2009). Además presenta nociones de un aprendizaje cooperativo, incidiendo en uno de los cinco pilares que Johnson, Johnson y Holubec (1999) nos destacan: interdependencia positiva.

La necesidad inherente de un contenido curricular como la enfermedad del Alzheimer, y los datos de 30 millones de personas en el mundo que padecen esta enfermedad, según la Organización Mundial de la Salud (OMS), a la vez que varios alumnos de sexto curso se interesaron por ella, fueron algunos de los detonantes para adentrarnos en esta experiencia educativa.

\section{Contextualización}

El proyecto interdisciplinar se lleva a cabo desde el área de Ciencias de la Naturaleza, el área de Lengua Castellana y Literatura y desde la de Matemáticas con los dos grupos de alumnos de sexto curso (24 alumnos en la clase A y 23 alumnos en la clase B). 
Todo comenzó cuando surgió el emergente en clase por parte de una alumna, sobre aspectos de esta enfermedad del sistema nervioso. Fue una clara oportunidad para aprovechar ciertos contenidos de las citadas áreas: la publicidad, expresión escrita, expresión oral, enfermedad del sistema nervioso, además de otros como las operaciones con los números decimales, al calcular la recaudación de la campaña.

La organización de la clase fue en grupos cooperativos no formales de cuatro alumnos, organización recomendada por los hermanos Johnson (2014). La connotación de no formal, como sendos autores indican, está relacionada con la temporalidad de la misma, siendo esta únicamente para la realización del presente proyecto. Cada miembro dentro del grupo tenía un rol (responsable, ayudante del responsable, secretario y portavoz) tal y como apuntan los hermanos Johnson (2014), que debía cumplir diariamente, materializándose los avances del grupo en un cuaderno de seguimiento intragrupal que más adelante se expondrá.

\section{Diseño y desarrollo}

El proyecto fue diseñado para doce días incluyendo la visita a la Asociación de Familiares de Alzheimer de la Ribera (en adelante AFAR) pudiendo obtener más información en: http://afaranda.com/

El trabajo giró en torno a un índice diseñado para iniciarse en el método científico. En él podíamos ver los siguientes epígrafes: introducción, síntomas, cuidados, diseño de la camiseta, conclusiones, bibliografía y anexos.

La línea de aprendizaje que se siguió fue en relación a unos folletos y el libro Cómo hablar con un enfermo de Alzheimer de Stauss (2014)que nos proporcionó la citada asociación. Además el portavoz del grupo, podía levantarse a “pedir cita” en el horario del ordenador que disponía el aula, para buscar información sobre los epígrafes del proyecto o bien entrar en la web: www.crowdence.espara crear el diseño de su camiseta, consensuada por el grupo. Esta aplicación fue enseñada días antes del proyecto, utilizando el cañón del aula, para proyectarla y explicar las diferentes pestañas que la conformaban y su uso.

Antes de comenzar el proyecto, se les recordó la forma en la que se les iba a evaluar, presentándoles la escala de evaluación, extraída del Grupo Actitudes, que ya conocían de antemano debido a la sistematicidad de la misma. 
ESCALA DE VALORACIÓN DEL TRABAJO COOPERATIVO|

\begin{tabular}{|c|c|c|c|c|c|}
\hline CRITERIOS & \multicolumn{4}{|c|}{ DESCRIPTORES } & TOTAL \\
\hline $\begin{array}{c}\text { Cooperación } \\
\text { (30) }\end{array}$ & $\begin{array}{l}\text { No nos hemos } \\
\text { puesto de acuerdo } \\
\text { en múltiples } \\
\text { ocasiones } \\
\text { (5) }\end{array}$ & \multicolumn{2}{|c|}{$\begin{array}{l}\text { Nos hemos puesto de acuerdo, pero con un gran } \\
\text { esfuerzo } \\
(10)\end{array}$} & $\begin{array}{l}\text { Hemos compartido ideas y } \\
\text { hemos llegado a acuerdos } \\
\text { comunes sin ningún enfado } \\
\text { (30) }\end{array}$ & \\
\hline $\begin{array}{l}\text { Ortografia } \\
\qquad(20)\end{array}$ & $\begin{array}{l}\text { El trabajo tiene más } \\
\text { de } 8 \text { faltas de } \\
\text { ortografía } \\
(0)\end{array}$ & \begin{tabular}{||c||} 
El trabajo tiene entre \\
$6-7$ faltas de ortografía \\
(5)
\end{tabular} & \begin{tabular}{||c||} 
El trabajo tiene entre 3.5 \\
faltas de ortografía
\end{tabular} & $\begin{array}{c}\text { El trabajo tiene menos de } 2 \text { faltas } \\
\text { de ortografía } \\
(20)\end{array}$ & \\
\hline $\begin{array}{c}\text { Contenidos } \\
\text { (30) }\end{array}$ & $\begin{array}{l}\text { El trabajo no recoge } \\
\text { ninguno de los } \\
\text { contenidos } \\
\text { solicitados. } \\
(0)\end{array}$ & \begin{tabular}{|} 
El trabajo no recoge \\
alguno de los \\
contenidos solicitados \\
(5)
\end{tabular} & \begin{tabular}{|} 
El trabajo recoge todos los \\
contenidos solicitados, \\
aunque de manera \\
confusa. \\
$(20)$
\end{tabular} & $\begin{array}{l}\text { El trabajo recoge todos los } \\
\text { contenidos solicitados y de una } \\
\text { forma ordenada y clara } \\
(30)\end{array}$ & \\
\hline $\begin{array}{c}\text { Exposición } \\
(20)\end{array}$ & $\begin{array}{l}\text { Para la exposición ha } \\
\text { sido necesario leer el } \\
\text { trabajo } \\
(0)\end{array}$ & \multicolumn{2}{|c|}{\begin{tabular}{|l} 
La exposición es autónoma sin necesidad de leer \\
el trabajo \\
$(10)$
\end{tabular}} & $\begin{array}{l}\text { La exposición es natural y se } \\
\text { disfruta exponiendo nuestro } \\
\text { trabajo } \\
(20)\end{array}$ & \\
\hline PUNTOS TO & TALES & & & & $/ 10$ \\
\hline
\end{tabular}

Tabla 1.

Escala de valoración del trabajo cooperativo

Una de las partes del proyecto, fue la visita a AFAR, dividiendo a ambos sextos, por temas organizativos de la asociación. La visita se preparó previamente, con la coordinadora de AFAR. Desde el centro preparamos una entrevista con preguntas; contenido del bloque temático: comunicación oral, hablar y escuchar, como por ejemplo: ¿qué es lo que más te gusta de AFAR? ¿cuántos hijos tienes? con el fin de interactuar con los usuarios y ver el grado de afectación que podían presentar. Además de las estrategias lúdicas que allí trabajamos, cabe destacar la actividad de canoterapia, con un perro adiestrado profesionalmente para interactuar con los usuarios, dándoles tarjetas, jugando con ellos... actividad muy enriquecedora sendos públicos.

Finalizado ya el proyecto, solo nos quedaba exponerlo, utilizando la técnica cooperativa de cabezas numeradas que los hermanos Johnson (2014) nos apuntan, donde un alumno de cada grupo sería el encargado de exponer al resto de compañeros el trabajo de su equipo, teniendo en cuenta una serie de ítems que se le iban a evaluarno calificar y que,sabían previamente, al hacerles entrega de la siguiente lista de control extraída del Grupo Actitudes (Tabla 2).

Una vez expuestos todos los trabajos con sus respectivos diseños de camisetas, nos juntamos ambos sextospara decidir de forma democráticael diseño de la camiseta 
que lanzaríamos a campaña real, exhibiendo todas las fotografías de las camisetas y votando el que ellos personalmente creían que iba a tener mayor repercusión

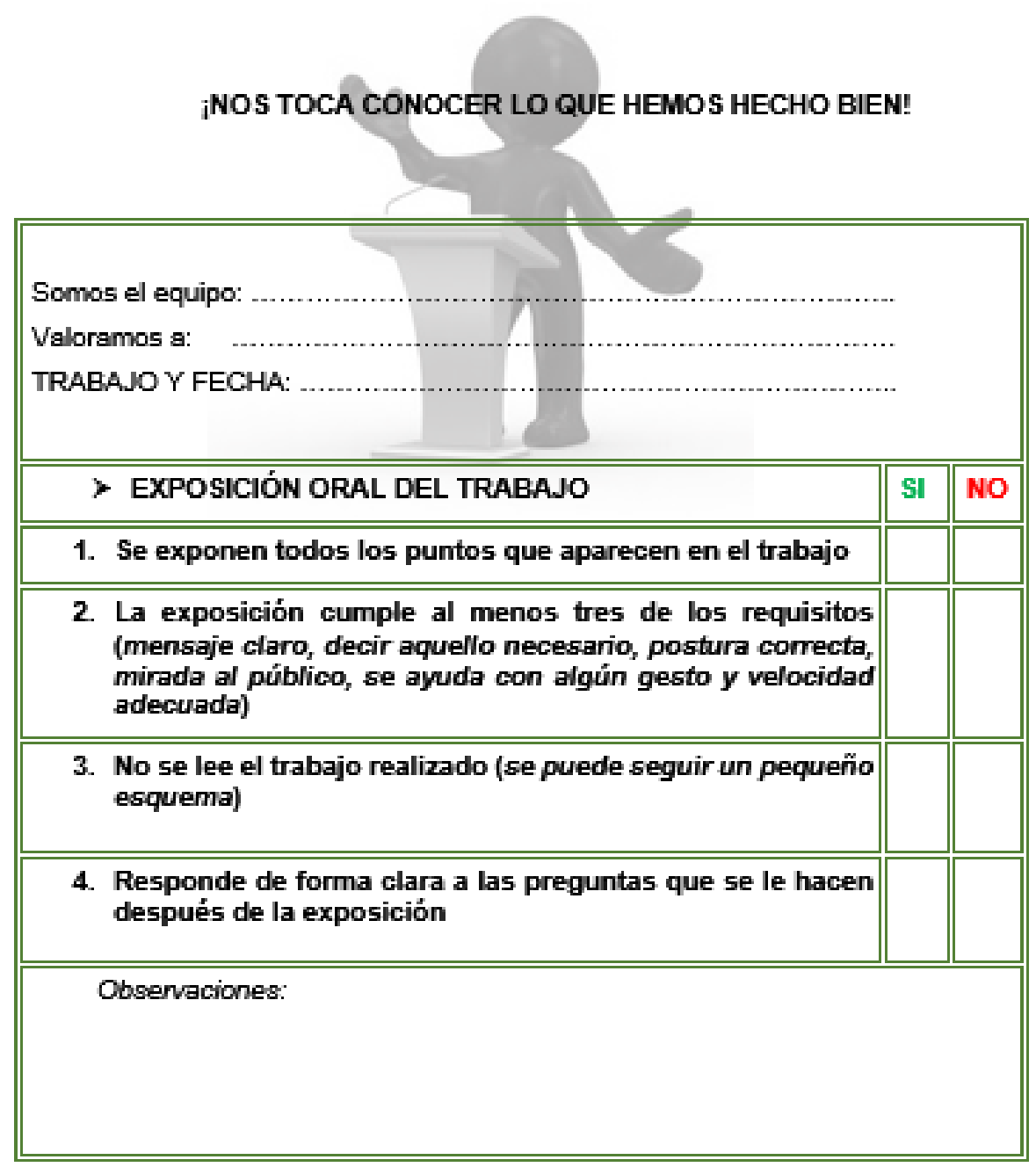

Tabla 2.

Lista de control para las exposiciones orales

\section{Evaluación y conclusiones}

La actividad de evaluación final consistió en que cada grupo debía repartir los puntos conseguidos en función de la puntuación total de su escala.Esta, debía ser dividida entre los integrantes del equipo, utilizando la objetividad que nos proporcionada el cuaderno de seguimiento intragrupal, extraído del Grupo Actitudes, donde el secretario del grupo en consenso con todos los demás, sitúa a cada alumno en uno de los aspectos a valorar de forma diaria, con el fin formativo de la mejora del trabajo 


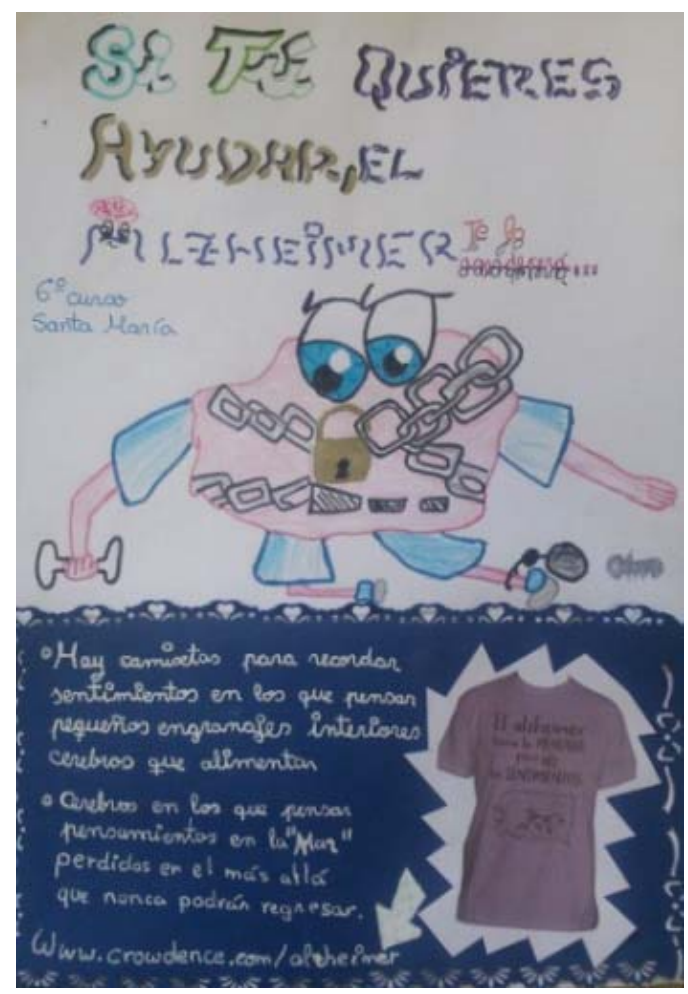

Figura 1.

Póster para publicitar la campaña por la localidad.

CUADERNO DE SEGUMMENTO INTRAGRUPAL

Llevar un recuento de los pequeños grandes pasos que dais en el día.

UN PEQUEÑO PASO PARA VOSOTROS, UN GRAN PASO PARA EL TRABAJO.

* Solo os podéís colocar en la casilla que más corresponda con vuestro trabajo diario, siempre consensuado con los compañeros.



Tabla 3.

Cuaderno de seguimiento intragrupal

El Alzheimer borra la memoria pero no los sentimientos 
Para terminar el proyecto, señalar que el reparto de camisetas también fue gestionado por los propios alumnos. Crowdence nos envió cuatro cajas con 393 camisetas, y unos listados por nombre y número de pedido, que debían ir tachando los propios alumnos a medida que las personas venían a recoger sus camisetas. La recaudación total fue de 1407,06 euros, destinados a AFAR.

\section{Referencias}

Fernández Enguita, M. (1997). La escuela a examen. Un análisis sociológico para educadores y otras personas interesadas. Madrid: Pirámide.

Johnson, D., Johnson, R., \&Holubec, E. (1999). El aprendizaje cooperativo en el aula. Buenos Aires: Paidós.

Johnson, D. W., \& Johnson, R. T. (2014). La evaluación en el aprendizaje cooperativo: cómo mejorar la evaluación individual a través del grupo. Madrid: SM

Puig, J. M. (2009). Aprendizaje servicio (APS). Educación y compromiso cívico. Graó. Barcelona.

Strauss, C. J. (2014). Cómo hablar con un enfermo de Alzheimer. Madrid: Obelisco. 